EUROPEAN JOURNAL OF PURE AND APPLIED MATHEMATICS

Vol. 14, No. 4, 2021, 1415-1428

ISSN 1307-5543 - ejpam.com

Published by New York Business Global

\title{
Revisiting Domination, Hop Domination, and Global Hop Domination in Graphs
}

\author{
Gemma Salasalan ${ }^{1, *}$, Sergio R. Canoy, Jr. ${ }^{2,3}$ \\ 1 Department of Arts and Sciences, Institute of Teacher Education, Arts and Sciences, \\ Davao del Sur State College, Matti, Digos City, Davao del Sur, Philippines \\ 2 Department of Mathematics and Statistics, College of Science and Mathematics, \\ MSU-Iligan Institute of Technology, , 9200 Iligan City, Philippines \\ 3 Center of Graph Theory, Algebra and Analysis, Premier Research Institute of Science and \\ Mathematics, MSU-Iligan Institute of Technology, 9200 Iligan City, Philippines
}

\begin{abstract}
A set $S \subseteq V(G)$ is a hop dominating set of $G$ if for each $v \in V(G) \backslash S$, there exists $w \in S$ such that $d_{G}(v, w)=2$. It is a global hop dominating set of $G$ if it is a hop dominating set of both $G$ and the complement $\bar{G}$ of $G$. The minimum cardinality of a hop dominating (global hop dominating) set of $G$, denoted by $\gamma_{h}(G)$ (resp. $\gamma_{g h}(G)$ ), is called the hop domination (resp. global hop domination) number of $G$. In this paper, we give some realization results involving domination, hop domination, and global hop domination parameters. Also, we give a rectification of a result found in a recent paper of the authors and use this to prove some results in this paper.
\end{abstract}

2020 Mathematics Subject Classifications: 05C69

Key Words and Phrases: Domination, hop domination, global hop domination, complementary prism, shadow graph

\section{Introduction}

Domination has been a topic of interest to many researchers in the field of Graph Theory. By imposing certain additional conditions or formulating similar conditions from the standard concept, a variant can then be obtained. Indeed, the domination concept yielded several variations which have been investigated by researchers. Some of these variants can be found in [1], [2], [3], [4], [6], [7], [8], [9], [10] and [14].

Shortly after Natarajan and Ayyaswamy [13] introduced and studied the concept of hop domination in a graph, some variants of the concept emerged. The concept and some of its variants are studied in [5], [11], [12], [15], and [16]. In this paper, we show that the standard domination and hop domination parameters are generally non-comparable. It

${ }^{*}$ Corresponding author.

DOI: https://doi.org/10.29020/nybg.ejpam.v14i4.4144

Email addresses: gemma.salasalan@g.msuiit.edu.ph (G. Salasalan),

sergio.canoy@g.msuitt.edu.ph (S. Canoy, Jr.) 
is shown that the absolute difference of these parameters can be made arbitrarily large. Further, we rectify a result found in [16] and use the corrected result to prove some results in this paper.

Let $G=(V(G), E(G))$ be a simple undirected graph. The distance between two vertices $u$ and $v$ of $G$, denoted by $d_{G}(u, v)$, is equal to the length of a shortest path connecting $u$ and $v$. Any path connecting $u$ and $v$ of length $d_{G}(u, v)$ is called a $u-v$ geodesic. The open neighbourhood of a vertex $v$ of $G$ is the set $N_{G}(v)=\{u \in V(G): u v \in E(G)\}$ and its closed neighbourhood is the set $N_{G}[v]=N_{G}(v) \cup\{v\}$. The open neighbourhood of a subset $S$ of $V(G)$ is the set $N_{G}(S)=\cup_{v \in S} N_{G}(v)$ and its closed neighbourhood is the set $N_{G}[S]=N_{G}(S) \cup S$. The degree of $v$, denoted by $\operatorname{deg}_{G}(v)$, is equal to $\left|N_{G}(v)\right|$. A vertex $v$ is called a leaf in $G$ if $\operatorname{deg}_{G}(v)=1$. The open hop neighbourhood of a vertex $v$ of $G$ is the set $N_{G}(v, 2)=\left\{w \in V(G): d_{G}(v, w)=2\right\}$ and its closed hop neighbourhood is the set $N_{G}[v, 2]=N_{G}(v, 2) \cup\{v\}$. The open hop neighbourhood of a subset $S$ of $V(G)$ is the set $N_{G}(S, 2)=\cup_{v \in S} N_{G}(v, 2)$ and its closed hop neighbourhood is the set $N_{G}[S, 2]=$ $N_{G}(S, 2) \cup S$.

A set $S \subseteq V(G)$ is a dominating set of $G$ if $N_{G}[S]=V(G)$. A vertex $v$ of $G$ is a dominating vertex if $\{v\}$ is a dominating set of $G$. The smallest cardinality of a dominating set of $G$, denoted by $\gamma(G)$, is called the domination number of $G$. A dominating set of $G$ with with cardinality $\gamma(G)$ is called a $\gamma$-set of $G$.

A set $S \subseteq V(G)$ is a hop dominating set of $G$ if for each $x \in V(G) \backslash S$, there exists $z \in S$ such that $d_{G}(x, z)=2$. The smallest cardinality of a hop dominating set of $G$, denoted by $\gamma_{h}(G)$, is called the hop domination number of $G$. A hop dominating set of $G$ with cardinality $\gamma_{h}(G)$ is called a $\gamma_{h}$-set of $G$. A set $S \subseteq V(G)$ is a global hop dominating set of $G$ if it is a hop dominating set of $G$ and $\bar{G}$. The smallest cardinality of a global hop dominating set of $G$, denoted by $\gamma_{g h}(G)$, is called the global hop domination number of $G$. A global hop dominating set of $G$ with cardinality $\gamma_{g h}(G)$ is called a $\gamma_{g h}$-set of $G$.

\section{Results}

We note that although hop domination is, in some sense, a variation of the standard domination concept, the associated parameters are, in general, not comparable. Our first simple result says that the absolute difference of the domination number and hop domination number can be made arbitrarily large.

Proposition 1. Each of the following statements holds.

(i) For each integer $n \geq 1$, there exists a connected graph $G$ such that $\gamma_{h}(G)-\gamma(G)=n$.

(ii) For each integer $n \geq 1$, there exists a connected graph $G$ such that $\gamma(G)-\gamma_{h}(G)=n$.

Proof. (i) Let $G=K_{n+1}$. Then $\gamma(G)=1$ and $\gamma_{h}(G)=n+1$. Hence, $\gamma_{h}(G)-\gamma(G)=n$.

(ii) Consider the star $K_{1, n+2}$ with vertices $v_{0}, v_{1}, v_{2}, \ldots, v_{n+1}, v_{n+2}$, where $v_{0}$ is the central vertex. Let $G$ (see Figure 1 ) be the graph obtained from $K_{1, n+2}$ by adding $n+2$ pendant edges $v_{1} w_{1}, v_{2} w_{2}, \ldots, v_{n+1} w_{n+1}, v_{n+2} w_{n+2}$. Let $S_{1}=\left\{v_{1}, v_{2}, \ldots, v_{n}, v_{n+1}, v_{n+2}\right\}$ 


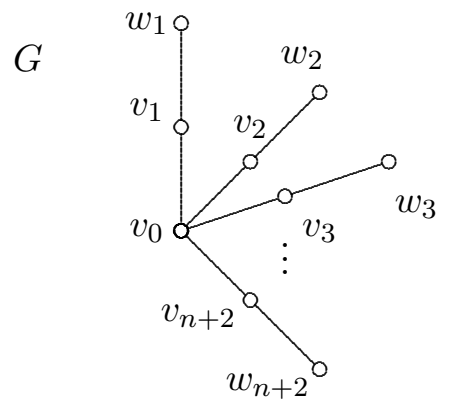

Figure 1

and $S_{2}=\left\{v_{0}, v_{1}\right\}$. Clearly, $S_{1}$ and $S_{2}$ are $\gamma$-set and $\gamma_{h}$-set of $G$, respectively. Thus, $\gamma(G)-\gamma_{h}(G)=(n+2)-2=n$.

The next result is, in fact, a realization problem.

Theorem 1. Let $a$ and $b$ be positive integers. Then each of the following statements holds.

(i) If $2 \leq a \leq b$, then there exists a connected graph $G$ such that $\gamma_{h}(G)=a$ and $\gamma(G)=b$.

(ii) If $3 \leq a \leq b$, then there exists a graph $H$ such that $\gamma(H)=a$ and $\gamma_{h}(H)=b$.

Proof. (i) Suppose first that $a=b$. Consider the graph $G$ in Figure 2. Clearly, $S_{1}=\left\{x_{1}, x_{2}, \ldots, x_{a}\right\}$ is a $\gamma_{h}$-set and $S_{2}=\left\{y_{1}, y_{2}, \ldots, y_{a}\right\}$ is a $\gamma$-set of $G$. Hence, $\gamma_{h}(G)=$ $\gamma(G)=a$.

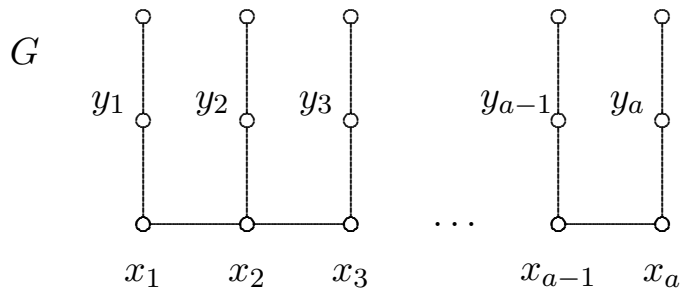

Figure 2

Next, suppose $a<b$ and let $m=b-a$. Consider the graph $G$ in Figure 3. One can easily see that set $S=\left\{x_{1}, x_{2}, \ldots, x_{a}\right\}$ is a $\gamma_{h}$-set of $G$. Hence, $\gamma_{h}(G)=|S|=a$. The set $S^{\prime}=\left\{y_{1}, y_{2}, \ldots, y_{a}, z_{1}, z_{2}, \ldots, z_{m}\right\}$ is a $\gamma$-set of $G$ and so $\gamma(G)=\left|S^{\prime}\right|=a+m=b$. 

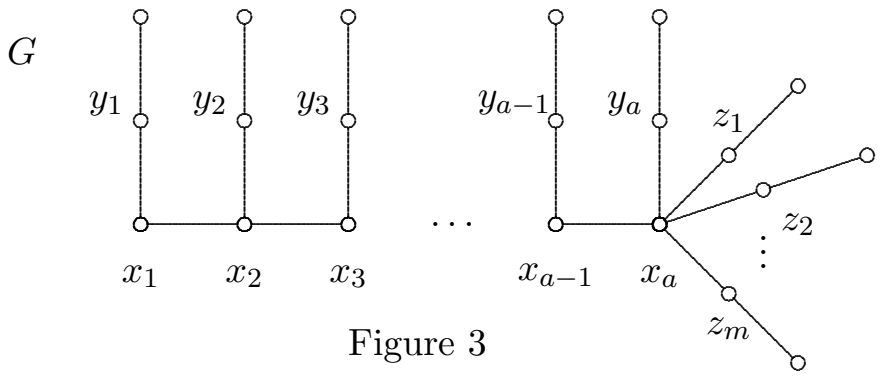

(ii) The case $a=b$ is similar to the first case of $(i)$. Suppose $a<b$ and let $m=b-a$. Consider graph $H=G \cup K_{m+1}$, where $V\left(K_{m+1}\right)=\left\{v_{1}, v_{2}, \ldots, v_{m}, v_{m+1}\right\}$ and $G$ is the graph in Figure 4. Then $S=\left\{y_{1}, y_{2}, \ldots, y_{a-1}, v_{1}\right\}$ is a $\gamma$-set and $S^{\prime}=\left\{x_{1}, x_{2}, \ldots, x_{a-1}\right\} \cup$

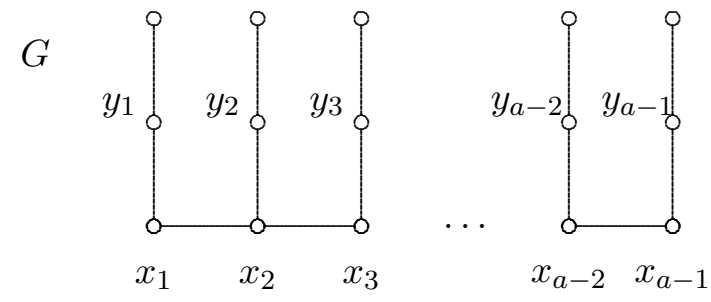

Figure 4

$V\left(K_{m+1}\right)$ is a $\gamma_{h}$-set of $H$. Therefore, $\gamma(H)=a$ and $\gamma_{h}(H)=a-1+m+1=a+m=b$.

It must be clear that every global hop dominating set is a hop dominating a set and a set is a global hop dominating set of a graph $G$ if and only if it is a global hop dominating set of $\bar{G}$. The following remark is immediate from these facts.

Remark 1. For any graph $G, \gamma_{h}(G) \leq \gamma_{g h}(G)$ and $\gamma_{g h}(G)=\gamma_{g h}(\bar{G})$.

It was pointed out in [16] that $1 \leq \gamma_{g h}(G) \leq|V(G)|$ for any graph $G$ and that $\gamma_{g h}(G)=1$ if and only if $G=K_{1}$. The next result is a rectification of Theorem 3.3 in [16].

Theorem 2. (Theorem 3.3 in [16]) Let $G$ be a graph of order $n \geq 1$. Then $\gamma_{g h}(G)=n$ if and only if every component of $G$ or $\bar{G}$ is complete. Moreover, if $G$ is connected, then for each $v \in V(G)$, we have

(i) $V(G) \backslash N_{G}(v)$ is an independent set, and

(ii) $N_{G}(v)=N_{G}(a)$ for each $a \in V(G) \backslash N_{G}(v)$.

Proof. Suppose $\gamma_{g h}(G)=n$. Assume first that $G$ is disconnected and suppose that $G$ has a component $C$ which is not complete. Then there exist distinct vertices $x, y \in V(C)$ such that $d_{G}(x, y)=d_{C}(x, y)=2$. Let $S=V(G) \backslash\{x\}$. Then $S$ is a hop dominating set of $G$. Let $z \in C$ such that $[x, z, y]$ is an $x$-y geodesic in $G$. Let $C^{\prime}$ be a component of 
$G$ with $C^{\prime} \neq C$ and pick any $w \in C^{\prime}$. Then $[x, w, z]$ is an $x$ - $z$ geodesic in $\bar{G}$. It follows that $d_{\bar{G}}(x, z)=2$. Thus, $S$ is a hop dominating set of $\bar{G}$, showing that $S$ is a global hop dominating set of $\bar{G}$. Therefore, $\gamma_{g h}(G) \leq|S|=n-1$, a contradiction. Accordingly, every component of $G$ is complete.

Next, suppose that $G$ is connected. Suppose further that $\bar{G}$ is connected. Then, clearly, $G \neq K_{n}$. Let $u, v \in V(G)$ be such that $d_{G}(u, v)=2$ and let $[u, p, v]$ be a $u-v$ geodesic in $G$. Then $S^{*}=V(G) \backslash\{u\}$ is a hop dominating set of $G$. Since $u p \notin E(\bar{G})$, it follows that $d_{\bar{G}}(u, p) \geq 2$. It follows that there exists $q \in S$ such that $d_{\bar{G}}(u, q)=2$. This shows that $S^{*}$ is hop dominating set of $\bar{G}$. Thus, $S^{*}$ is a global hop dominating set of $G$ and $\gamma_{g h}(G) \leq\left|S^{*}\right|=n-1$, a contradiction. Therefore, $\bar{G}$ is disconnected. Since $\gamma_{g h}(\bar{G})=\gamma_{g h}(G)=n$, this would imply that every component of $\bar{G}$ is complete (as in the first case applied to $\bar{G})$.

For the converse, suppose first that every component of $G$ is complete. Then, clearly, $S=V(G)$ is the only hop dominating set of $G$. It follows that $S$ is the only global hop dominating set of $G$. If every component of $\bar{G}$ is complete, then $S$ is the only global hop dominating set of $G$. Therefore, $\gamma_{g h}(G)=n$.

Now suppose $G$ is connected and let $v \in V(G)=V(\bar{G})$. Suppose there exist distinct vertices $a, b \in V(G) \backslash N_{G}(v)$ such that $a b \in E(G)$. Then $[a, v, b]$ is an $a$-b geodesic in $\bar{G}$, implying that $S_{a}=V(G) \backslash\{a\}$ is a hop dominating set of $\bar{G}$. Now, since $a \in V(G) \backslash N_{G}(v)$, it follows that $d_{G}(a, v) \geq 2$. This implies that there exists $w \in S_{a}$ such that $d_{G}(a, w)=2$, showing that $S_{a}$ is also a hop dominating set of $G$. Hence, $\gamma_{g h}(G) \leq\left|S_{a}\right|=n-1$, a contradiction. Therefore, $V(G) \backslash N_{G}(v)$ is an independent set, showing that $(i)$ holds. Next, let $a \in V(G) \backslash N_{G}(v)$. Let $C_{v}$ be the component of $\bar{G}$ with $v \in C_{v}$. Since $a \in N_{\bar{G}}(v)$ and $C_{v}$ is complete, $N_{G}(a)=N_{G}(v)$. This shows that (ii) holds.

The next result is a consequence of Theorem 2 .

Corollary 1. $\gamma_{g h}\left(K_{n}\right)=\gamma_{g h}\left(K_{1, n-1}\right)=n$ for all integer $n \geq 2$.

Theorem 3. Let $a$ and $b$ be positive integers such that $2 \leq a \leq b$. Then there exists $a$ connected graph $G$ such that $\gamma_{h}(G)=a$ and $\gamma_{g h}(G)=b$.

Proof. Consider the following cases:

Case 1. $a=b$

Let $G=K_{a}$. Then $\bar{G}=\bar{K}_{a}$. By Theorem $2, \gamma_{h}(G)=\gamma_{g h}(G)=a$.

Case 2. $a<b$

Let $k=b-a$. Let $V\left(K_{a-1}\right)=\left\{x_{1}, x_{2}, \ldots, x_{a-1}\right\}$ and consider the graph $G$ in Figure 5 obtained from $\langle v\rangle+K_{a-1}$ by adding the edges $x_{i} y_{j}$ for $i \in\{1,2, \ldots, a-1\}$ and $j \in$ $\{1,2, \ldots, k\}(\langle v\rangle$ is the graph induced by $\{v\})$. 


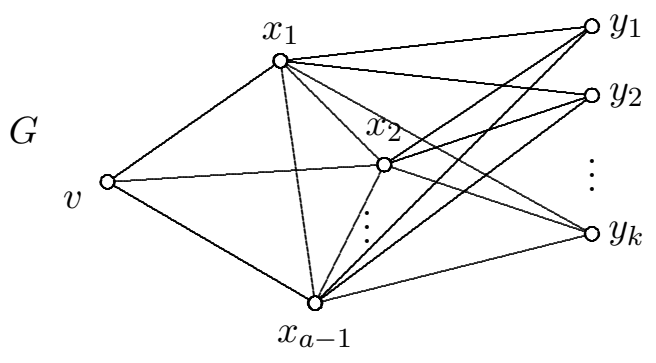

Figure 5

Let $S=\{v\} \cup V\left(K_{a-1}\right)=\left\{v, x_{1}, x_{2}, \ldots, x_{a-1}\right\}$. Since every vertex $x_{i}(1 \leq i \leq a-1)$ is a dominating vertex of $G$, it follows that each $x_{i}$ is in every $\gamma_{h}$-set of $G$. Since $d_{G}\left(v, y_{j}\right)=2$ for all $j \in\{1,2, \ldots, k\}$, it follows that $S$ is a $\gamma_{h}$-set of $G$. Hence, $\gamma_{h}(G)=a$. Now, the complement $\bar{G}$ of $G$ is the graph isomorphic to $\left(K_{1}+K_{k}\right) \cup \bar{K}_{a-1}$. By Theorem 2, $\gamma_{g h}(G)=|V(G)|=(k+1)+(a-1)=b$.

Corollary 2. For each positive integer $n$, there exists a connected graph $G$ such that $\gamma_{g h}(G)-\gamma_{h}(G)=n$. In other words, the difference $\gamma_{g h}-\gamma_{h}$ can be made arbitrarily large.

Proof. Let $n$ be a positive integer. By Theorem 3, there exists a connected graph $G$ such that $\gamma_{h}(G)=n+1$ and $\gamma_{g h}(G)=2 n+1$. Hence, $\gamma_{g h}(G)-\gamma_{h}(G)=n$.

For a graph $G$, the complementary prism, denoted by $G \bar{G}$, is formed from the disjoint union of $G$ and its complement $\bar{G}$ by adding a perfect matching between corresponding vertices of $G$ and $\bar{G}$. For each $v \in V(G)$, let $\bar{v}$ denote the vertex corresponding to $v$ in $\bar{G}$. In simple terms, the graph $G \bar{G}$ is formed from $G \cup \bar{G}$ by adding the edge $v \bar{v}$ for every vertex $v \in V(G)$.

The next result gives bounds for the domination number of the complementary prism of a graph.

Theorem 4. [10] For any graph $G, \max \{\gamma(G), \gamma(\bar{G})\} \leq \gamma(G \bar{G}) \leq \gamma(G)+\gamma(\bar{G})$.

Theorem 5. Let $G$ be a connected graph of order $n$. Then each of following holds.

(i) If $G$ is a non-trivial graph such that $\gamma(G)=1$, then $\gamma(G \bar{G})=1+\gamma(\bar{G} \backslash \bar{v})$, where $v$ is a dominating vertex of $G$. In particular, $\gamma\left(K_{n} \bar{K}_{n}\right)=n$.

(ii) If $n \geq 1$, then $\gamma_{h}(G \bar{G})=2$. In particular, $\{v, \bar{v}\}$ is $\gamma_{h}$-set of $G \bar{G}$ for each $v \in V(G)$.

(iii) If $n \geq 2$, then $\gamma_{g h}(G \bar{G}) \leq \min \left\{n, 2 \gamma_{g h}(G)\right\}$.

Proof. (i) Let $v$ be a dominating vertex of $G$ and let $D$ be a dominating set of $\bar{G} \backslash \bar{v}$. Since $(V(G) \backslash\{v\}) \cup\{\bar{v}\} \subseteq N_{G \bar{G}}(v)$ and $V(\bar{G}) \backslash\{\bar{v}\} \subseteq N_{G \bar{G}}(D), S=D \cup\{v\}$ is a dominating set of $G \bar{G}$. This implies that $\gamma(G \bar{G}) \leq 1+|D|=1+\gamma(\bar{G} \backslash \bar{v})$. 
Suppose now that $S_{0}$ is a $\gamma$-set of $G \bar{G}$. Since $v$ is a dominating vertex of $G, \bar{v}$ is an isolated vertex of $\bar{G}$ (and so a leaf in $G \bar{G}$ ). Hence, $v \in S_{0}$ or $\bar{v} \in S_{0}$. Suppose $v \notin S_{0}$. Then $\bar{v} \in S_{0}$. Suppose $S_{1}=S_{0} \cap V(G)=\varnothing$. Since $N_{G \bar{G}}(w) \cap V(\bar{G})=\{\bar{w}\}$ for each $w \in V(G)$, it follows that $S_{0}=V(\bar{G})$. Hence, $\gamma(G \bar{G})=\left|S_{0}\right|=n \geq 1+\gamma(\bar{G} \backslash \bar{v})$. Suppose $S_{1} \neq \varnothing$ and let $S_{2}=S_{0} \cap(V(\bar{G}) \backslash\{\bar{v}\})$. If $S_{2}$ is a dominating of $\bar{G} \backslash \bar{v}$, then $\gamma(G \bar{G})=\left|S_{0}\right| \geq 1+\gamma(\bar{G} \backslash \bar{v})$. Suppose $R=V(\bar{G} \backslash \bar{v}) \backslash N_{\bar{G} \backslash \bar{v}}\left[S_{2}\right] \neq \varnothing$ and set $R_{G}=\{w \in V(G) \backslash\{v\}: \bar{w} \in R\}$. Then necessarily, $R_{G} \subseteq S_{1}$. Thus, $\gamma(G \bar{G})=\left|S_{0}\right|=1+\left|S_{1}\right|+\left|S_{2}\right| \geq 1+\left|R_{G}\right|+\left|S_{2}\right| \geq 1+\gamma(\bar{G} \backslash \bar{v})$.

Next, suppose that $v \in S_{0}$. Since $S_{0}$ is a $\gamma$-set of $G \bar{G}$ and $\bar{v}$ is a leaf of $G \bar{G}, \bar{v} \notin S$. Let $D_{1}=(V(G) \backslash\{v\}) \cap S_{0}$. If $D_{1}=\varnothing$, then $D_{2}=\left(V(\bar{G}) \cap S_{0}\right.$ must be a dominating set of $\bar{G} \backslash \bar{v}$. It follows that $\gamma(G \bar{G})=\left|S_{0}\right| \geq 1+\gamma(\bar{G} \backslash \bar{v})$. Suppose that $D_{1} \neq \varnothing$. If $D_{2}=\varnothing$, then $D_{1}=V(G) \backslash\{v\}$. It follows that $S_{0}=V(G)$ and $\gamma(G \bar{G})=\left|S_{0}\right|=n \geq 1+\gamma(\bar{G} \backslash \bar{v})$. Suppose $D_{2} \neq \varnothing$ and let $D_{2}^{*}=V(\bar{G} \backslash \bar{v}) \backslash N_{\bar{G} \backslash \bar{v}}\left[D_{2}\right]$.Since $S_{0}$ is a $\gamma$-set and $v \in S_{0}$, it follows that $D_{2}^{*}=\left\{\bar{x}: x \in D_{1}\right\}$ and $\left|D_{2}^{*}\right|=\left|D_{1}\right|$. Clearly, $D^{\prime}=D_{2} \cup D_{2}^{*}$ is a dominating set of $\bar{G} \backslash \bar{v}$ and so $\gamma(G \bar{G})=\left|S_{0}\right|=1+\left|D_{1}\right|+\left|D_{2}\right|=1+\left|D^{\prime}\right| \geq 1+\gamma(\bar{G} \backslash \bar{v})$. This proves the desired equality. Thus, in particular, if $G=K_{n}$, then $\gamma\left(K_{n} \overline{K_{n}}\right)=n$.

(ii) If $n=1$, then $G \bar{G}=K_{2}$. Hence, $\gamma_{h}(G \bar{G})=2$. Suppose $n \geq 2$. Let $v \in V(G)$ and let $S=\{v, \bar{v}\}$. Let $w \in V(G) \backslash\{v\}$. If $w v \in E(G)$, then $[w, v, \bar{v}]$ is a $w-\bar{v}$ geodesic in $G \bar{G}$. If $w v \notin E(G)$, then $\bar{w} \bar{v} \in E(\bar{G})$. It follows that $[w, \bar{w}, \bar{v}]$ is a $w-\bar{v}$ geodesic in $G \bar{G}$. Next, let $\bar{z} \in V(\bar{G}) \backslash\{\bar{v}\}$. If $\bar{z} \bar{v} \in E(\bar{G})$, then $[\bar{z}, \bar{v}, v]$ is a $\bar{z}-v$ geodesic in $G \bar{G}$. If $\bar{z} \bar{v} \notin E(\bar{G})$, then $z v \in E(G)$ and $[\bar{z}, z, v]$ is a $\bar{z}-v$ geodesic in $G \bar{G}$. Thus, $S$ is a hop dominating set of $G$. Since $G \bar{G}$ is non-trivial, it follows that $\gamma_{h}(G \bar{G})=2$.

(iii) Suppose $n \geq 2$. Let $S=V(G)$ and let $\bar{v} \in V(G \bar{G}) \backslash S=V(\bar{G})$. Since $G$ is connected and non-trivial, we may choose any $w \in V(G) \cap N_{G}(v)$. Consequently, $[\bar{v}, v, w]$ is a $\bar{v}$ - $w$ geodesic in $G \bar{G}$. Hence, $d_{G \bar{G}}(\bar{v}, w)=2$. Since $\bar{v}$ was arbitrarily chosen, it follows that $S=V(G)$ is a hop dominating set of $G \bar{G}$. Next, let $\bar{y} \in V(\overline{G \bar{G}}) \backslash S$. Then $y \bar{y} \notin E(\overline{G \bar{G}})$. Pick any $x \in V(G) \backslash\{y\}$. If $x y \in E(G \bar{G})$, then $\bar{x} \bar{y} \in E(\overline{G \bar{G}})$. Since $y \bar{x} \in E(\overline{G \bar{G}})$, it follows that $d_{\overline{G \bar{G}}}(\bar{y}, y)=2$. If $x y \notin E(G \bar{G})$, then $x y \in E(\overline{G \bar{G}})$. Since $x \bar{y} \in E(\overline{G \bar{G}})$, $d \overline{G \bar{G}}(\bar{y}, y)=2$. This shows that $S$ is also a hop dominating set of $\overline{G \bar{G}}$. Therefore, $S$ is a global hop dominating set of $G \bar{G}$ and $\gamma_{g h}(G \bar{G}) \leq|S|=n$.

Now let $S_{G}$ be a global hop dominating set of $G$ and let $S_{\bar{G}}=\left\{\bar{v}: v \in S_{G}\right\}$. Then clearly, $S^{\prime}=S_{G} \cup S_{\bar{G}}$ is a hop dominating set of both $G \bar{G}$ and $\overline{G \bar{G}}$, that is, $S^{\prime}$ is a global hop dominating set of $G \bar{G}$. Thus, in particular, if $S_{G}$ is a $\gamma_{g h}$-set of $G$, then $S^{\prime}=S_{G} \cup S_{\bar{G}}$ is a global hop dominating set of $G \bar{G}$. This implies that $\gamma_{g h}(G \bar{G}) \leq\left|S^{\prime}\right|=2 \gamma_{g h}(G)$. Combining this with the first inequality, we find that the assertion in (iii) holds.

The bound given in Theorem 5(iii) is sharp. To see this, consider the graph $G=P_{3}$ and the graph $H$ obtained from $C_{4}$ by adding a pendant edge. It can be verified that $\gamma_{g h}(G \bar{G})=|V(G)|=3$ and $\gamma_{g h}(H \bar{H})=2 \gamma_{g h}(H)=4<5=|V(H)|$.

Theorem 6. Let $G=K_{m_{1}, m_{2}, \ldots, m_{k}}$ be a complete multipartite graph such that $m_{1} \leq m_{2} \leq$ 
$\ldots \leq m_{k}$ and $k \geq 2$. Then

$$
\gamma(G \bar{G})= \begin{cases}k & \text { if } m_{1}=1 \\ k+1 & \text { if } m_{1}=2 \\ k+2 & \text { if } m_{1} \geq 3\end{cases}
$$

Proof. Let $U_{1}, U_{2}, \ldots, U_{k}$ be the partite sets of $G$ with $\left|U_{i}\right|=m_{i}$ for each $i \in$ $\{1,2, \ldots, k\}$. For each $i \in\{1,2, \ldots, k\}$, let $\bar{U}_{i}=\left\{\bar{v}: v \in U_{i}\right\}$. Then the induced graphs of the sets $\bar{U}_{1}, \bar{U}_{2}, \ldots, \bar{U}_{k}$ are exactly the (complete) components of $\bar{G}$ in $G \bar{G}$. Suppose first that $m_{1}=1$, say $U_{1}=\{v\}$. Then $v$ is a dominating vertex of $G$ and so by Theorem $5(i), \gamma(G \bar{G})=1+\gamma(\bar{G} \backslash \bar{v})$. Since $\bar{G} \backslash \bar{v}$ is the disjoint union of complete graphs $\left\langle\bar{U}_{2}\right\rangle,\left\langle\bar{U}_{3}\right\rangle, \ldots,\left\langle\bar{U}_{k}\right\rangle$, it follows that $\gamma(\bar{G} \backslash \bar{v})=k-1$. Thus, $\gamma(G \bar{G})=k$.

Next, suppose that $m_{1}=2$, say $U_{1}=\{u, v\}$. By Theorem $4, \max \{\gamma(G), \gamma(\bar{G})\}=$ $k \leq \gamma(G \bar{G}) \leq k+2=\gamma(G)+\gamma(\bar{G})$. For each $i \in\{2 \ldots, k\}$, choose any $v_{i} \in U_{i}$ and let $S=\{u, \bar{v}\} \cup\left\{\bar{v}_{i}: i \in\{2,3, \ldots, k\}\right\}$. Then $S$ is a dominating set of $G \bar{G}$. Hence, $\gamma(G \bar{G}) \leq|S|=2+k-1=k+1$. Let $S^{\prime}$ be a $\gamma$-set of $G \bar{G}$. If $S^{\prime} \cap V(\bar{G})=\varnothing$ or $S^{\prime} \cap V(G)=\varnothing$, then $\left|S^{\prime}\right|=|V(G)|=\sum_{i=1}^{k} m_{i} \geq 2 k>k+1$ which is not possible. Thus, $S^{\prime} \cap V(\bar{G}) \neq \varnothing$ and $S^{\prime} \cap V(G) \neq \varnothing$. Clearly, $S^{\prime} \cap\left(U_{i} \cup \bar{U}_{i}\right) \neq \varnothing$ for all $i \in\{1,2, \ldots, k\}$. Suppose $S^{\prime} \cap U_{1} \neq \varnothing$. If $\left|S^{\prime} \cap U_{1}\right|=1$, say $v \in S^{\prime} \cap U_{1}$, then $V(G) \backslash\{u, v\} \subseteq N_{G \bar{G}}(v)$. Since $S^{\prime}$ is a $\gamma$-set of $G \bar{G}$ and $u \notin S^{\prime},\left|S^{\prime} \cap \bar{U}_{1}\right|=1$. We may assume that $\bar{u} \in S^{\prime}$. Let $j \in\{2,3, \ldots, k\}$ and suppose that $S \cap \bar{U}_{j}=\varnothing$. Then necessarily, $U_{j} \subseteq S^{\prime}$. Pick any $\bar{w} \in \bar{U}_{j}$ and let $S_{w}=\left(S^{\prime} \backslash U_{j}\right) \cup\{\bar{w}\}$. Then $S_{w}$ is a dominating set of $G \bar{G}$. Since $\left|U_{j}\right| \geq 2$, $\gamma(G \bar{G})=\left|S^{\prime}\right|>\left|S_{w}\right|$, a contradiction. Therefore, $S^{\prime} \cap \bar{U}_{j} \neq \varnothing$ for each $j \in\{2,3, \ldots, k\}$. Moreover, because $S^{\prime}$ is a $\gamma$-set of $G \bar{G},\left|S^{\prime} \cap \bar{U}_{j}\right|=1$ for all $j \in\{2,3, \ldots, k\}$. Thus, $\gamma(G \bar{G})=\left|S^{\prime}\right| \geq k+1$. If $\left|S^{\prime} \cap U_{1}\right|=2$, then $S^{\prime} \cap \bar{U}_{1}=\varnothing$. Since $S^{\prime} \cap\left(U_{i} \cup \bar{U}_{i}\right) \neq \varnothing$ for all $i \in\{2, \ldots, k\}$, it follows that $\gamma(G \bar{G})=\left|S^{\prime}\right| \geq k+1$. Suppose now that $S^{\prime} \cap U_{1}=\varnothing$. If $\left|S^{\prime} \cap \bar{U}_{1}\right|=1$, then there exists $j \neq 1$ such that $S^{\prime} \cap U_{j} \neq \varnothing$. If $\left|S^{\prime} \cap U_{j}\right|=1$, then $\left|S^{\prime} \cap \bar{U}_{j}\right| \neq 0$. Since $S^{\prime} \cap\left(U_{i} \cup \bar{U}_{i}\right) \neq \varnothing$ for all $i \in\{2, \ldots, j-1, j+2, \ldots, k\}$, it follows that $\gamma(G \bar{G})=\left|S^{\prime}\right| \geq k+1$. If $\left|S^{\prime} \cap U_{j}\right| \geq 2$, then clearly, $\gamma(G \bar{G})=\left|S^{\prime}\right| \geq k+1$. Therefore, $\gamma(G \bar{G})=k+1$.

Finally, let $m_{1} \geq 3$. Let $S$ be a $\gamma$-set of $G \bar{G}$. Since $\gamma(G \bar{G}) \leq k+2$ (by Theorem 4) and $|V(G)|=\sum_{i=1}^{k} m_{i}>k+2, S \cap V(\bar{G}) \neq \varnothing$ and $S \cap V(G) \neq \varnothing$. Again, $S \cap\left(U_{i} \cup \bar{U}_{i}\right) \neq \varnothing$ for each $i \in\{1,2, \ldots, k\}$. Suppose $S \cap U_{1}=\varnothing$. Then $S \cap \bar{U}_{1} \neq \varnothing$. If $\left|S \cap \bar{U}_{1}\right| \geq 3$, then $\gamma(G \bar{G})=|S| \geq k+2$. So suppose that $\left|S \cap \bar{U}_{1}\right| \leq 2$. Then there exists $j \neq 1$ such that $S \cap U_{j} \neq \varnothing$. If $S \cap U_{j}=U_{j}$, then $\gamma(G \bar{G})=|S| \geq k+2$. If $S \cap U_{j} \neq U_{j}$, then $S \cap \bar{U}_{j} \neq \varnothing$. Hence, if $\left|S \cap \bar{U}_{1}\right|=2$, then $\gamma(G \bar{G})=|S| \geq k+2$. Suppose now that $\left|S \cap \bar{U}_{1}\right|=1$. Suppose further that $\left|S \cap\left(U_{j} \cup \bar{U}_{j}\right)\right|=2$. Then there exists $r \neq 1, j$ such that $\left|S \cap\left(U_{r} \cup \bar{U}_{r}\right)\right| \geq 2$. Thus, $\gamma(G \bar{G})=|S| \geq k+2$.

Next, suppose that $S \cap U_{1} \neq \varnothing$. Suppose $S \cap U_{j}=\varnothing$ for all $j \geq 2$. Since $S$ is a $\gamma$-set of $G \bar{G}$ and $U_{j} \subseteq N_{G \bar{G}}\left(S \cap U_{1}\right)$ for all $j \geq 2,\left|S \cap \bar{U}_{j}\right|=1$ for all $j \geq 2$ and $|S|=k-1+\left|U_{1}\right|$. If $m_{1}=\left|U_{1}\right|=3$, then $|S|=k-1+3=k+2$. However, if $m_{1} \geq 4$, then $|S|=k-1+\left|U_{1}\right| \geq k+3$, contrary to the fact that $k+2$ is an upper bound of $|S|$. Hence, for $m_{1} \geq 4$, there exists $j \geq 2$ such that $\left|S \cap\left(U_{j} \cup \bar{U}_{j}\right)\right| \geq 2$. Since $\left|S \cap\left(U_{1} \cup \bar{U}_{1}\right)\right| \geq 2, S$ 
is a $\gamma$-set, $\gamma(G \bar{G})=|S| \geq k+2$. Note that we obtain the same implications if $m_{1}=3$ and $S \cap U_{j} \neq \varnothing$ for some $j \geq 2$.

Accordingly, $\gamma(G \bar{G})=k+2$.

Given a graph $G$ with $\gamma(G)=1$, we denote by $\operatorname{Dom}(G)$ the set $\{v \in V(G)$ : $\{v\}$ is a dominating set of $G\}$.

Lemma 1. Let $G$ be a graph with $\gamma(G)=1$ and let $S$ be a global hop dominating set of $G \bar{G}$. If $v \in \operatorname{Dom}(G)$, then $v \in S$ or $\bar{v} \in S$.

Proof. Let $v \in \operatorname{Dom}(G)$ and suppose that $v, \bar{v} \notin S$. Since $S$ is a global hop dominating set of $G \bar{G}$, it is a hop dominating set of $\overline{G \bar{G}}$. As $\bar{v} \in V(\overline{G \bar{G}}) \backslash S$, there exists $z \in S$ such that $d_{\overline{G \bar{G}}}(\bar{v}, z)=2$. However, $d_{\overline{G \bar{G}}}(\bar{v}, x)=1$ for all $x \in V(\overline{G \bar{G}}) \backslash\{v, \bar{v}\}$. Since $v \notin S$, it follows that such a vertex $z$ does not exist, contrary to the assumption that $S$ is a hop dominating set of of $\overline{G \bar{G}}$. Therefore, $v \in S$ or $\bar{v} \in S$.

Corollary 3. For each positive integer $n \geq 2, \gamma_{g h}\left(K_{n} \bar{K}_{n}\right)=n$.

Proof. By Theorem 5(iii), $\gamma_{g h}\left(K_{n} \bar{K}_{n}\right) \leq n$. Let $S$ be a $\gamma_{g h}$-set of $K_{n} \bar{K}_{n}$. Since $\operatorname{Dom}\left(K_{n}\right)=V\left(K_{n}\right), \gamma_{g h}\left(K_{n} \bar{K}_{n}\right)=|S| \geq n$ by Lemma 1 . Therefore, $\gamma_{g h}\left(K_{n} \bar{K}_{n}\right)=n$.

Theorem 7. Let $G=K_{m_{1}, m_{2}, \ldots, m_{k}}$ be a complete multipartite graph such that $1 \leq m_{1} \leq$ $m_{2} \leq \ldots \leq m_{k}$, where $k \geq 2$ and $m_{j} \geq 2$ for some $j$ with $1 \leq j \leq k$. Then

$$
\gamma_{g h}(G \bar{G})= \begin{cases}k & \text { if } m_{1}=m_{2}=1 \text { and } k \geq 4 \\ k+1 & \text { otherwise. }\end{cases}
$$

Proof. Let $U_{1}, U_{2}, \ldots, U_{k}$ be the partite sets of $G$ with $\left|U_{i}\right|=m_{i}$ for each $i \in$ $\{1,2, \ldots, k\}$. Again, for each $i \in\{1,2, \ldots, k\}$, let $\bar{U}_{i}=\left\{\bar{v}: v \in U_{i}\right\}$. Choose any $v_{i} \in U_{i}$ for each $i \in\{1,2, \ldots, k\}$. Suppose $m_{1}=m_{2}=1$ and $k \geq 4$. Then $U_{1}=\left\{v_{1}\right\}$ and $U_{2}=\left\{v_{2}\right\}$. Let $S=\left\{v_{1}, v_{2}, \overline{v_{3}}, \overline{v_{4}}, \ldots, \overline{v_{k}}\right\}$. Then $d_{G \bar{G}}\left(\overline{v_{1}}, v_{2}\right)=2$ and $d_{G \bar{G}}\left(\overline{v_{2}}, v_{1}\right)=2$. For each $j \in\{3,4, \ldots, k\}$, we have $d_{G \bar{G}}\left(\bar{w}, v_{1}\right)=2$ for each $\bar{w} \in \overline{U_{j}} \backslash S$ and $d_{G \bar{G}}\left(x, \overline{v_{r}}\right)=2$ for each $x \in U_{j}$, where $r \geq 3$ and $r \neq j$. Thus, $S$ is a hop dominating set of $G \bar{G}$. On the other hand, $d_{\overline{G \bar{G}}}\left(\overline{v_{1}}, v_{1}\right)=2$ and $d_{\overline{G \bar{G}}}\left(\overline{v_{2}}, v_{2}\right)=2$. For each $j \in\{3,4, \ldots, k\}$, we have $d_{\overline{G \bar{G}}}\left(\bar{w}, \overline{v_{j}}\right)=2$ for each each $\bar{w} \in \overline{U_{j}} \backslash S$ and $d_{\overline{G \bar{G}}}\left(x, v_{1}\right)=2$ for each each $x \in U_{j}$. Thus, $S$ is a hop dominating set of $\overline{G \bar{G}}$. Therefore, $S$ is a global hop dominating set of $G \bar{G}$ and $\gamma_{g h}(G \bar{G}) \leq|S|=k$.

Next, let $S_{0}$ be a $\gamma_{g h}$-set of $G \bar{G}$. Suppose there exists $j \in\{1,2, \ldots, k\}$ such that $S_{0} \cap\left(U_{j} \cup \bar{U}_{j}\right)=\varnothing$. Let $\bar{w} \in \bar{U}_{j}$. It follows from the adjacency in $\overline{G \bar{G}}$ that $d_{\overline{G \bar{G}}}(\bar{w}, p)=1$ for all $p \in V(\overline{G \bar{G}}) \backslash\left(\bar{U}_{j} \cup\{w\}\right)$. Hence, by assumption, there exists no $q \in S_{0}$ with $d_{\overline{G \bar{G}}}(\bar{w}, q)=$ 2 , contrary to the fact that $S_{0}$ is a hop dominating set of $\overline{G \bar{G}}$. Therefore, $S_{0} \cap\left(U_{j} \cup \bar{U}_{j}\right) \neq \varnothing$ for each $j \in\{1,2, \ldots, k\}$. Consequently, $\gamma_{g h}(G \bar{G})=|S| \geq k$. Accordingly, $\gamma_{g h}(G \bar{G})=k$. 
Suppose now that the conditions $m_{1}=m_{2}=1$ and $k \geq 4$ do not hold. Let $S^{\prime}=$ $\left\{v_{1}, \bar{v}_{1}, \bar{v}_{2}, \bar{v}_{3}, \ldots, \bar{v}_{k}\right\}$. By Theorem $5(i i),\left\{v_{1}, \bar{v}_{1}\right\}$ is a hop dominating set of $G \bar{G}$. Hence, $S$ is a hop dominating set of $G \bar{G}$.

Let $v^{\prime} \in U_{j} \backslash\left\{v_{1}\right\}$, where $j \in\{1,2, \ldots, k\}$. Then $\left[v^{\prime}, \bar{v}_{1}, \bar{v}_{2}\right]$ is a $v^{\prime}-\bar{v}_{2}$ geodesic in $\overline{G \bar{G}}$. Hence, $d_{\overline{G \bar{G}}}\left(v^{\prime}, \bar{v}_{2}\right)=2$. Let $j \in\{1,2, \ldots, k\}$ and pick any $i \in\{1,2, \ldots, k\} \backslash\{j\}$. Then for $\bar{y} \in \bar{U}_{j} \backslash\left\{\bar{v}_{j}\right\}$, we find that $\left[\bar{y}, \bar{v}_{i}, \bar{v}_{j}\right]$ is a $\bar{y}-\bar{v}_{j}$ geodesic in $\overline{G \bar{G}}$. Hence, $d_{\overline{G \bar{G}}}\left(\bar{y}, \bar{v}_{j}\right)=2$. This shows that $S^{\prime}$ is a hop dominating set of $\overline{G \bar{G}}$. Therefore, $S^{\prime}$ is a global hop dominating set of $G \bar{G}$ and $\gamma_{g h}(G \bar{G}) \leq\left|S^{\prime}\right|=k+1$.

Let $S_{0}$ be a $\gamma_{g h}$-set of $G \bar{G}$. As shown and seen earlier $S_{0} \cap\left(U_{j} \cup \bar{U}_{j}\right) \neq \varnothing$ for each $j \in\{1,2, \ldots, k\}$. Next, suppose there exists $j \in\{1,2, \ldots, k\}$ with $m_{j} \geq 2$ such that $\left|S_{0} \cap \bar{U}_{j}\right|=0$. Let $\bar{a} \in \bar{U}_{j}$. Since $d_{\overline{G \bar{G}}}(\bar{a}, p)=1$ for $p \in V(\overline{G \bar{G}}) \backslash\left(\bar{U}_{j} \cup\{a\}\right)$ and $\left|S_{0} \cap \bar{U}_{j}\right|=0$, it follows that $a \in S_{0}$. Thus, $U_{j} \subseteq S_{0}$. Since $m_{j} \geq 2$, it follows that $\gamma_{g h}(G \bar{G})=\left|S_{0}\right| \geq k+1$. Suppose that $\left|S_{0} \cap \bar{U}_{j}\right| \neq 0$ for each $j$ with $m_{j} \geq 2$. If $\left|S_{0} \cap \bar{U}_{j}\right| \geq 2$ for some $j$ with $m_{j} \geq 2$, then $\gamma_{g h}(G \bar{G})=\left|S_{0}\right| \geq k+1$. Suppose that $\left|S_{0} \cap \bar{U}_{j}\right|=1$ for each $j$ with $m_{j} \geq 2$. For a $j$ satisfying this property, pick $\bar{y} \in \bar{U}_{j} \backslash S_{0}$. Since $S$ is a hop dominating set of $G \bar{G}$ and the induced graph of $\bar{U}_{j}$ is a complete graph in $G \bar{G}$, it follows that there exists $r \neq j$ such $z \in U_{r} \cap S_{0}$ and $d_{G \bar{G}}(\bar{y}, z)=2$. If an $r$ exists such that $m_{r} \geq 2$, then this would imply that $\gamma_{g h}(G \bar{G})=\left|S_{0}\right| \geq k+1$. So suppose that there exists no such $r$ with $m_{r} \geq 2$. Then $m_{r}=1$ and $r=1$ or $r=2$, say $U_{r}=U_{1}$. If $\bar{z} \in S_{0}$, then $\gamma_{g h}(G \bar{G})=\left|S_{0}\right| \geq k+1$. Suppose $\bar{z} \notin S_{0}$. Then there exists $p \in U_{s} \cap S_{0}$ for some $s \geq 2$ such that $d_{G \bar{G}}(\bar{z}, p)=2$. If $m_{s}=1$, then $s=2$ and $j=k=3$ by assumption. If $\bar{p} \in S_{0}$, then $\gamma_{g h}(G \bar{G})=\left|S_{0}\right| \geq k+1$. Suppose $\bar{p} \notin S_{0}$ and let $S_{0} \cap \bar{U}_{j}=\{\bar{q}\}$. Since $S_{0}$ is a hop dominating set of $G \bar{G}, \bar{z}, \bar{p} \notin S_{0}$, and $k=3$, we must have $q \in S_{0}$. Hence, $\gamma_{g h}(G \bar{G})=\left|S_{0}\right| \geq k+1$. Now, if $m_{s} \geq 2$, then $s=k$ by assumption. This implies that $\left|S_{0} \cap\left(U_{j} \cup \bar{U}_{j}\right)\right| \geq 2$, showing that $\gamma_{g h}(G \bar{G})=\left|S_{0}\right| \geq k+1$. Therefore, $\gamma_{g h}(G \bar{G})=k+1$.

The shadow graph $D_{2}(G)$ of a graph $G$ is the graph obtained by taking two copies of $G$, say $G_{1}$ and $G_{2}$, and joining each vertex $u \in V\left(G_{1}\right)$ to the neighbors of the corresponding vertex $u^{\prime} \in V\left(G_{2}\right)$.

Lemma 2. Let $G$ be a non-trivial connected graph and let $G_{1}$ and $G_{2}$ be copies of $G$ in the graph $D_{2}(G)$. If $w \in V\left(G_{1}\right)$ and $w^{\prime} \in V\left(G_{2}\right)$ is the corresponding vertex of $w$, then

$$
N_{D_{2}(G)}[w, 2]=N_{G_{1}}[w, 2] \cup N_{G_{2}}\left[w^{\prime}, 2\right]=N_{D_{2}(G)}\left[w^{\prime}, 2\right] .
$$

Proof. Clearly, $N_{G_{1}}[w, 2] \cup N_{G_{2}}\left[w^{\prime}, 2\right] \subseteq N_{D_{2}(G)}[w, 2]$. Now let $x \in N_{D_{2}(G)}[w, 2]$. Then $x=w$ or $d_{D_{2}(G)}(w, x)=2$. Suppose first that $x \in V\left(G_{1}\right)$. If $x=w$, then $x \in N_{G_{1}}[w, 2]$. Suppose that $d_{D_{2}(G)}(w, x)=2$ and let $y \in V\left(D_{2}(G)\right)$ such that $[w, y, x]$ is $w$-x geodesic in $D_{2}(G)$. If $y \in V\left(G_{1}\right)$, then $[w, y, x]$ is a $w$-x geodesic in $G_{1}$. Suppose $y \in V\left(G_{2}\right)$, say $y=u^{\prime}$. By definition of $D_{2}(G)$, it follows that $u \in V\left(G_{1}\right)$ and $[w, u, x]$ is a $w$-x geodesic in $G_{1}$. Hence, $x \in N_{G_{1}}[w, 2]$. Next, suppose that $x=z^{\prime} \in V\left(G_{2}\right)$. If $y \in V\left(G_{1}\right)$, then $\left[w^{\prime}, y^{\prime}, z^{\prime}\right]$ is a $w$-x geodesic in $G_{2}$. Suppose $y \in V\left(G_{2}\right)$, say $y=u^{\prime}$. Then $\left[w^{\prime}, u^{\prime}, z^{\prime}\right]$ is $w^{\prime}-x$ geodesic in $G_{2}$. Hence, $x \in N_{G_{2}}\left[w^{\prime}, 2\right]$. Thus, $N_{D_{2}(G)}[w, 2] \subseteq N_{G_{1}}[w, 2] \cup N_{G_{2}}\left[w^{\prime}, 2\right]$, showing that 
$N_{D_{2}(G)}[w, 2] \subseteq N_{G_{1}}[w, 2] \cup N_{G_{2}}\left[w^{\prime}, 2\right]$. Similarly, $N_{D_{2}(G)}\left[w^{\prime}, 2\right] \subseteq N_{G_{1}}[w, 2] \cup N_{G_{2}}\left[w^{\prime}, 2\right]$. Therefore, $N_{D_{2}(G)}[w, 2]=N_{G_{1}}[w, 2] \cup N_{G_{2}}\left[w^{\prime}, 2\right]=N_{D_{2}(G)}\left[w^{\prime}, 2\right]$.

A result in [13] says that $\gamma_{h}\left(D_{2}(G)\right)=\gamma_{h}(G)$ for any graph $G$. This, however, is not true if $G$ contains an isolated vertex. Indeed, if $G$ is the trivial graph and $H$ is the (disjoint) union $K_{1} \cup K_{1} \cup P_{2}$, then $D_{2}(G)=\bar{K}_{2}$ and $D_{2}(G)=\bar{K}_{2} \cup \bar{K}_{2} \cup C_{4}$. Hence, $\gamma_{h}\left(D_{2}(G)\right)=2 \neq 1=\gamma_{h}(G)$ and $\gamma_{h}\left(D_{2}(H)\right)=6 \neq 4=\gamma_{h}(G)$.

Theorem 8. Let $G$ be a non-trivial graph. Then the following hold.

(i) If $G$ is connected, then $\gamma_{h}\left(D_{2}(G)\right)=\gamma_{h}(G)$.

(ii) If $G$ is disconnected with $r$ trivial components and $k$ non-trivial components $G_{1}, G_{2}$, $G_{3}, \ldots, G_{k}$, then $\gamma_{h}\left(D_{2}(G)\right)=2 r+\sum_{i=1}^{k} \gamma_{h}\left(G_{k}\right)$.

Proof. (i) Let $G_{1}$ and $G_{2}$ be the two copies of $G$ in the definition of $D_{2}(G)$. Let $S$ be a $\gamma_{h}$-set of $G_{1}$ and let $v^{\prime} \in V\left(G_{2}\right)$. If the corresponding vertex $v \in V\left(G_{1}\right)$ is in $S$, then $d_{D_{2}(G)}\left(v, v^{\prime}\right)=2$. So suppose $v \notin S$. Since $S$ is a hop dominating set of $G_{1}$, there exists $w \in S$ such that $d_{G_{1}}(v, w)=2$. Let $[v, z, w]$ be a $v$ - $w$ geodesic in $G_{1}$. Then $v^{\prime} z, z w \in E\left(D_{2}(G)\right)$. Since $v \notin N_{G_{1}}(w), v^{\prime} w \notin E\left(D_{2}(G)\right)$. Thus, $d_{D_{2}(G)}\left(w, v^{\prime}\right)=2$. Therefore, $S$ is a hop dominating set of $D_{2}(G)$ and $\gamma_{h}\left(D_{2}(G) \leq|S|=\gamma_{h}(G)\right.$.

Next, suppose that $S^{\prime}$ is a $\gamma_{h}$-set of $D_{2}(G)$. Let $S_{1}=S^{\prime} \cap V\left(G_{1}\right)$ and $S_{2}=S^{\prime} \cap V\left(G_{2}\right)$. If $S_{1}=S^{\prime}$ or $S_{2}=S^{\prime}$, then $S^{\prime}$ is a hop dominating set of $G_{1}$ or $G_{2}$. Hence, $\gamma_{h}\left(D_{2}(G)=\right.$ $\left|S^{\prime}\right| \geq \gamma_{h}(G)$. Suppose $S_{1} \neq \varnothing$ and $S_{2} \neq \varnothing$. If $S_{1}$ is a hop dominating set of $G_{1}$ or $S_{2}$ is a hop dominating set of $G_{2}$, then, as seen earlier, $S_{1}$ or $S_{2}$ is a hop dominating set of $D_{2}(G)$, contrary to the assumption that $S^{\prime}$ is a $\gamma_{h}$-set of $D_{2}(G)$. Hence, none of these two sets is a hop dominating set. Let $D_{G}=\left\{v \in V\left(G_{1}\right) \backslash S_{1}: v \notin N_{G_{1}}\left(S_{1}, 2\right)\right\}=V\left(G_{1}\right) \backslash N_{G_{1}}\left[S_{1}, 2\right]$ and let $S_{G}=\left\{v \in V\left(G_{1}\right) \backslash S_{1}: v^{\prime} \in S_{2}\right\}$. Clearly, if $v \in S_{G}$, then $v^{\prime} \in S_{2}$. Now let $y^{\prime} \in S_{2}$. Then $N_{D_{2}(G)}\left[y^{\prime}, 2\right]=N_{D_{2}(G)}[y, 2]$ by Lemma 2 . Since $S^{\prime}$ is a $\gamma_{h^{-}}$set of $D_{2}(G)$, it follows that $y \in V\left(G_{1}\right) \backslash S_{1}$, that is, $y \in S_{G}$. Hence, $\left|S_{G}\right|=\left|S_{2}\right|$. Since $S^{\prime}$ is a hop dominating set of $D_{2}(G), D_{G} \subseteq N_{D_{2}(G)}\left[S_{2}, 2\right]$. This means that if $w \in D_{G}$, then there exists $z^{\prime} \in S_{2}$ such that $w \in N_{D_{2}(G)}\left[z^{\prime}, 2\right]$. Consequently, $z \in S_{G}$ and by Lemma 2, we have $w \in N_{D_{2}(G)}[z, 2]=N_{G_{1}}[z, 2]$. Thus, $D_{G} \subseteq N_{G_{1}}\left[S_{G}, 2\right]$, showing that $S_{1} \cup S_{G}$ is a hop dominating set of $G_{1}$. Therefore, $\gamma_{h}(G)=\gamma_{h}\left(G_{1}\right) \leq\left|S_{1} \cup S_{G}\right|=\gamma_{h}\left(D_{2}(G)\right)$. This establishes the desired equality.

(ii) This follows from $(i)$ and the fact that the hop domination of a (disconnected) graph is the sum of the hop domination numbers of its components.

Theorem 9. Let $G$ be a non-trivial connected graph. Then $\gamma_{g h}\left(D_{2}(G)\right) \leq 2 \gamma_{g h}(G)$.

Proof. Let $G_{1}$ and $G_{2}$ be the two copies of $G$ in the definition of $D_{2}(G)$. Let $S_{1}$ be a $\gamma_{g h}$-set of $G_{1}$ and let $S_{2}=\left\{v^{\prime} \in V\left(G_{2}\right): v \in S_{1}\right\}$. Then $S_{2}$ is a $\gamma_{g h}$-set of $G_{2}$. Hence, $S=S_{1} \cup S_{2}$ is a hop dominating set of $D_{2}(G)$. Since $S_{1}$ and $S_{2}$ are also $\gamma_{g h}$-sets of $\bar{G}_{1}$ and $\bar{G}_{2}$, respectively, it follows that $S=S_{1} \cup S_{2}$ is a hop dominating set of $\overline{D_{2}(G)}$. Hence, $S=S_{1} \cup S_{2}$ is a global hop dominating set of $D_{2}(G)$ and $\gamma_{g h}\left(D_{2}(G)\right) \leq|S|=2 \gamma_{g h}(G)$.

The next result is easy. 
Lemma 3. Let $G$ be a non-trivial graph. Then each of the following statements is true.

(i) $D_{2}(G)$ is not a complete graph.

(ii) $D_{2}(G)$ is connected if and only if $G$ is connected.

Lemma 4. Let $G$ be a graph of order $n$. Then each of the following statements is true.

(i) Every component of $D_{2}(G)$ is a complete graph if and only if $G=\bar{K}_{n}$.

(ii) Every component of $\overline{D_{2}(G)}$ is a complete graph if and only if $G=\bar{K}_{n}$ or $G=K_{m_{1}, m_{2}, \ldots, m_{k}}$, where $\sum_{i=1}^{k} m_{i}=n$.

Proof. Let $G_{1}$ and $G_{2}$ be the two copies of $G$ in the definition of $D_{2}(G)$.

(i) Suppose every component of $D_{2}(G)$ is a complete graph. Suppose further that $G$ has a non-trivial component $H$. Then $D_{2}(H)$ is a component of $D_{2}(G)$ which is not complete by Lemma 3, a contradiction to our assumption of $D_{2}(G)$. Therefore, every component of $G$ is trivial, i.e., $G=\bar{K}_{n}$. The converse is clear.

(ii) Suppose that every component of $\overline{D_{2}(G)}$ is a complete graph. If $\overline{D_{2}(G)}$ is connected, then $D_{2}(G)=\bar{K}_{2 n}$. Hence, $G=\bar{K}_{n}$. Next, suppose that $\overline{D_{2}(G)}$ is disconnected with components $C_{1}, C_{2}, \ldots, C_{k}$. For each $i \in\{1,2, \ldots, k\}$, let $S_{1, i}=V\left(G_{1}\right) \cap V\left(C_{i}\right), S_{2, i}=$ $V\left(G_{2}\right) \cap V\left(C_{i}\right)$ and $m_{i}=\left|S_{1, i}\right|$. Note that $v \in S_{1, i}$ if and only if $v^{\prime} \in S_{2, i}$ and that $C_{i}=\left\langle S_{1, i} \cup S_{2, i}\right\rangle$ for each $i \in\{1,2, \ldots, k\}$. Let $i, j \in\{1,2, \ldots, k\}$ with $i \neq j$. Since $C_{i}$ and $C_{j}$ are complete subgraphs (components) of $\overline{D_{2}(G)}$, it follows that in graph $D_{2}(G), S_{1, i}$ and $S_{1, j}$ are independent subsets of $V\left(G_{1}\right)$ and $x y \in E\left(G_{1}\right)$ for each $x \in S_{1, i}$ and $y \in S_{1, j}$. It follows that $G_{1}$ is a complete multipartite graph with partite sets $S_{1,1}, S_{1,2}, \ldots, S_{1, k}$. Hence, $G=K_{m_{1}, m_{2}, \ldots, m_{k}}$.

The converse is clear.

Theorem 10. Let $G$ be a graph of order $n$. Then $\gamma_{g h}\left(D_{2}(G)\right)=2 n$ if and only if $G=\bar{K}_{n}$ or $G=K_{m_{1}, m_{2}, \ldots, m_{k}}$, where $\sum_{i=1}^{k} m_{i}=n$.

Proof. By Theorem 2, $\gamma_{g h}\left(D_{2}(G)\right)=2 n$ if and only if every component of $D_{2}(G)$ or $\overline{D_{2}(G)}$ is complete. Thus by Lemma $4, \gamma_{g h}\left(D_{2}(G)\right)=2 n$ if and only if $G=\bar{K}_{n}$ or $G=K_{m_{1}, m_{2}, \ldots, m_{k}}$, where $\sum_{i=1}^{k} m_{i}=n$.

Note that Theorem 10 shows that the bound given in Theorem 9 is tight.

Conclusion: The domination and hop domination parameters are, in general, not comparable. However, a result shows that the absolute difference of the domination number and hop domination number can be made arbitrarily large. On the other hand, a result shows a relationship of the hop domination and global hop domination numbers. 
For any non-trivial connected graph $G$, it is proved that that $2 \gamma_{g h}(G)$ is a tight bound for the global hop domination number of the shadow graph $D_{2}(G)$ of $G$. The authors are still unable to show that the strict inequality in Theorem 9 is also attainable. We leave to the interested readers to verify whether or not equality in this result holds.

\section{Acknowledgements}

The authors would like to thank the referees for reading the initial manuscript and the invaluable comments and suggestion they have given. Also, the authors are grateful to the Department of Science and Technology - Accelerated Science and Technology and Human Resource Development Program (DOST-ASTHRDP), Philippines, and MSU-Iligan Institute of Technology for funding this research.

\section{References}

[1] B. Arriola and S. Jr. Canoy. Secure doubly connected domination in graphs. International Journal of Mathematical Analysis, 8:1571-1580, 2014.

[2] A. Cabaro, S. Jr. Canoy, and I. Aniversario. Secure connected domination in a graph. International Journal of Mathematical Analysis, 8(42):2065-2074, 2014.

[3] S. Jr. Canoy, S.A. Canoy, and M. Cruzate. Global domination in a graph. Advances and Applications in Discrete Mathematics, 19(4):401-408, 2018.

[4] S. Jr. Canoy and G. Malacas. Determining the intruder's location in a given network: Locating-dominating sets in a graph. NRCP Research Journal., 13(1):1-8, 2013.

[5] S. Jr. Canoy, R. Mollejon, and J.G. Canoy. Hop dominating sets in graphs under binary operations. European Journal of Pure and Applied Mathematics, 12(4):14551463, 2019.

[6] H. Escuardo, R. Gera, A. Hansberg, A.J. Rad, and L. Volkman. Geodetic domination in graphs. Journal of Combinatorial Mathematics and Combinatorial Computing, 77:89-101, 2011.

[7] T. Haynes, S. Hedetniemi, and P. Slater. Domination in Graphs, Advanced Topics. Marcell Dekker, New York, USA, 1998.

[8] T. Haynes, S. Hedetniemi, and P. Slater. Fundamentals of Domination in Graphs. Marcell Dekker, New York, USA, 1998.

[9] T. Haynes, M. Henning, and J. Howard. Locating and total dominating sets in trees. Discrete Applied Mathematics, 154(8):1293-1300, 2006.

[10] T. Haynes, M. Henning, and L. van der Merwe. Domination and total domination in complementary prisms. Journal of Combinatorial Optimization, 18:23-37, 2009. 
[11] M. Henning and N.J. Rad. On 2-step and hop dominating sets in graphs. Graphs and Combinatorics, 33(4):913-927, 2017.

[12] G. Mahadevan and V. Vijayalakshmi. Clone hop domination number of a graph. Journal of Discrete Mathematical Sciences and Cryptography, 22(5):719-729, 2019.

[13] C. Natarajan and S. Ayyaswamy. Hop domination in graphs II. Versita, 23(2):187199, 2015.

[14] B. Omamalin, S. Jr. Canoy, and H. Rara. Locating total dominating sets in the join, corona, and composition of graphs. Applied Mathematical Sciences, 8(48):2363-2374, 2014 .

[15] Y. Pabilona and H. Rara. Connected hop domination in graphs under some binary operations. Asian-European Journal of Mathematics, 11(5):1850075-1-1850075-11, 2018.

[16] G. Salasalan and S. Jr. Canoy. Global hop domination numbers of graphs. European Journal of Pure and Applied Mathematics, 14(1):112-125, 2021. 